\title{
Comparison of efficacy of metformin and oral contraceptive combination of ethinyl estradiol and drospirenone in polycystic ovary syndrome
}

\author{
Yashasvi Suvarna ${ }^{1}$, Nivedita Maity ${ }^{1}$, Pramila Kalra ${ }^{2}$, MC Shivamurthy ${ }^{1}$ \\ ${ }^{\prime}$ Department of Pharmacology, M.S. Ramaiah Medical College and Hospitals, Karnataka, India \\ ${ }^{2}$ Department of Endocrinology, M.S. Ramaiah Medical College and Hospitals, Karnataka, India
}

\section{Abstract}

Objective: The 2013 Endocrine Society guidelines state that hormonal contraceptives should be used for treating both menstrual irregularity and hirsutism in patients with polycystic ovary syndrome (PCOS). Metformin should be reserved for the treatment of women presenting with only menstrual irregularity because it has limited benefits in treating hyperandrogenism associated with PCOS. A high prevalence of insulin resistance is noted among the South Asians, and these guidelines may not hold good for this population. Thus, this study was conducted to investigate and compare the effects of metformin and an oral contraceptive containing drospirenone on menstrual pattern, body mass index, serum testosterone levels, and dehydroepiandrosterone sulfate (DHEAS) levels at baseline to 6 months of therapy in the treatment groups.

Material and Methods: This was a prospective observational study that was conducted over a year in patients visiting the Endocrinology outpatient department at a tertiary care center in a south Indian city. Forty-six subjects diagnosed with PCOS as per the Rotterdam criteria were included. They received either metformin twice daily or an oral contraceptive containing drospirenone once daily as a monthly regimen for 6 months.

Results: Metformin regularized menstrual cycles in $72 \%$ of patients who were followed up at 6 months. No significant difference was observed between the two treatment groups with respect to decreasing the body mass index, serum testosterone levels, and DHEAS levels ( $p=0.40, p=0.65$, and $\mathrm{p}=0.22$, respectively).

Conclusion: Metformin is effective in regularizing menstrual cycles, decreasing body mass index, and treating hyperandrogenism in Indian women diagnosed with PCOS. (J Turk Ger Gynecol Assoc 2016; 17: 6-9)

Keywords: Polycystic ovary syndrome, metformin, oral contraceptive containing drospirenone, menstrual cycle, testosterone

Received: 14 July, $2015 \quad$ Accepted: 19 January, 2016

\section{Introduction}

Polycystic ovary syndrome (PCOS) is a common endocrine disorder with a prevalence of $15 \%-20 \%$ (1) and is characterized by oligomenorrhea, hyperandrogenism, and ultrasonographic evidence of PCOS (2).

Combined oral contraceptives have been the traditional choice for patients not desirous of conception (3) and patients who have a major component of hyperandrogenism. Metformin for treating insulin resistance has also been found to be beneficial in restoring ovulation in women suffering from PCOS (4), and thus, can be used as a therapeutic option. However, there are not many studies demonstrating its benefit in treating hyperandrogenism (5).

Guidelines released by the Endocrine Society state that metformin has limited or no benefit in treating hyperandrogenism and should be used if patients presenting with menstrual irregularity as the chief complaint (6). The applicability of these guidelines in South Asia, particularly India, is uncertain because of the high prevalence of insulin resistance amongst this population (7). The relevance of this observation concerning therapy for patients with PCOS is highlighted by a study where patients with PCOS of the South Asian ethnicity had more severe symptoms, higher fasting insulin concentrations, and lower insulin sensitivity compared with Caucasians (8). Thus, insulin-sensitizing drugs may have a more beneficial role to play in this population. Hence, this study was conducted to compare the efficacy of metformin and oral contraceptive containing drospirenone on menstrual pattern, body mass index (BMI), serum testosterone levels, and dehydroepiandrosterone sulfate (DHEAS) levels in Indian women with PCOS.

\section{Material and Methods}

This study was conducted at a tertiary care hospital in a south Indian city among patients visiting the Endocrinology outpatient department over a 1-year period. The study was approved by the Institutional Ethics Committee.

Patients aged between 18 and 45 years who were diagnosed with PCOS as per the Rotterdam criteria (2) with a modified 
Ferriman-Gallwey score of $>8$ were included. Patients with contraindications to oral contraceptives; patients desirous of conception within 6 months of inclusion in the study; patients with a diagnosis of concomitant hypothyroidism, hyperprolactinemia, diabetes mellitus, renal, or adrenal insufficiency; history of drug use for PCOS before inclusion in the study, and history of smoking and substance abuse were excluded from the study.

Forty-six patients were included, and informed consent was obtained. Demographic data, baseline parameters, such as menstrual pattern and BMI, were recorded. Baseline laboratory parameters, such as serum testosterone and DHEAS levels were recorded. The patients at the physician's discretion received either $30 \mu \mathrm{g}$ ethinyl estradiol $+3 \mathrm{mg}$ drospirenone (one tablet daily) as a monthly regimen of 21 days for 6 months or 2 $\mathrm{g}$ metformin sustained release tablet in two divided doses daily for 6 months.

The patients were followed up at 3 and 6 months of treatment, and changes in menstrual pattern, BMI, and serum androgen levels (testosterone and DHEAS) were recorded and compared between the two groups.

A priori sample size calculation was performed using a priori sample size calculator for Student's t-tests (software) version 3.0 (9) and considering an $\alpha$ value of $0.05, \beta$ value of 0.20 , and an anticipated effect size of 0.85 . The total sample size required was calculated to be 46 with 23 patients in each group. Student's t-test (two tailed, independent) was used to determine the significance of study parameters on a continuous scale between the two groups and chi-square test was used to determine the significance of study parameters on a categorical scale between the two groups. Student's t-test (two tailed, dependent) was used to determine the significance of study parameters on a continuous scale within each group. The analyses were performed using the Statistical Package for Social Sciences software version (SPSS) software version 17 (SPSS Inc.; Chicago, USA).

\section{Results}

Forty-six patients (23 in each group) were enrolled in the study, with $72 \%$ follow-up at 3 months and $48 \%$ follow-up at 6 months (Figure 1).

The baseline characteristics of patients in both the groups were comparable (Table 1). Regarding the menstrual pattern, nine of the 16 patients in the metformin group and all 17 patients who were analyzed in the oral contraceptive containing drospirenone group had attained regular menstrual cycles at the 3-month follow-up ( $p=0.002$ between the two groups), and eight of the 11 patients in the metformin group and all 11 patients in the oral contraceptive containing drospirenone group had attained regular menstrual cycles at the 6-month follow-up ( $p=0.06$ between the two groups). BMI, serum testosterone levels, and serum DHEAS levels significantly declined within the individual groups, but no significant difference was observed between the two groups at the 6-month follow-up (Table 2).

Both the drugs were well tolerated with no serious adverse events being recorded during the study period.

\section{Discussion}

Therapy for patients with PCOS is targeted toward regularization of menstrual cycles and suppression of ovarian testosterone production, which is conventionally, achieved using combined oral contraceptives. However, side effects, such as increased risk of venous thrombosis and weight gain, make the use of combined oral contraceptives inappropriate in obese women and women with prediabetes. Thus, alternative options are explored. Metformin is an insulin-sensitizing drug that has demonstrated promise in therapy for patients with PCOS. This study assessed the efficacy of metformin and oral contraceptive containing drospirenone in patients with PCOS.

All patients enrolled in the study had oligomenorrhea. As expected, oral contraceptive containing drospirenone regularized menstrual cycles in all patients. However, $72 \%$ of patients receiving metformin also had regular menstrual cycles after 6 months of therapy, with no significant difference being observed between

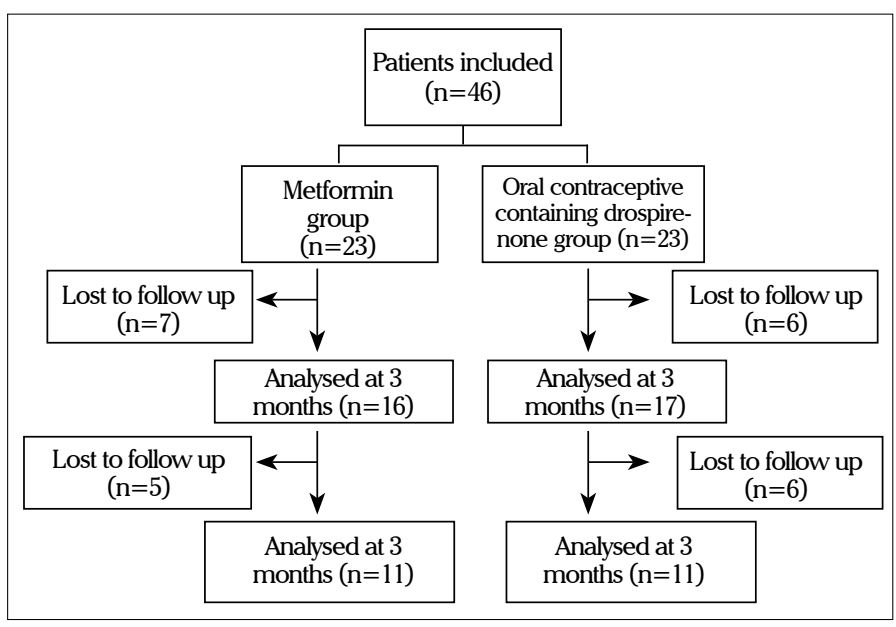

Figure 1. Patient recruitment chart

Table 1. Baseline characteristics of patients

\begin{tabular}{|l|c|c|c|}
\hline $\begin{array}{l}\text { Baseline } \\
\text { characteristics }\end{array}$ & $\begin{array}{c}\text { Metformin } \\
\text { group (n=23) }\end{array}$ & $\begin{array}{c}\text { Oral contraceptive } \\
\text { containing } \\
\text { drospirenone } \\
\text { group (n=23) }\end{array}$ & p \\
\hline Age (years) & $24.52 \pm 4.37$ & $23.52 \pm 6.04$ & 0.52 \\
\hline BMI (kg/m²) & $27.72 \pm 5.04$ & $26.48 \pm 4.56$ & 0.25 \\
\hline Patient symptoms and clinical signs \\
\hline 1. Oligomenorrhea & $23(100 \%)$ & $23(100 \%)$ & 1.00 \\
\hline $\begin{array}{l}\text { 2. Hirsutism } \\
\text { (FG score>8) }\end{array}$ & $23(100 \%)$ & $23(100 \%)$ & 1.00 \\
\hline 3. Infertility & $4(17.39 \%)$ & $2(8.69 \%)$ & 0.33 \\
\hline 4. Acne & $6(26.08 \%)$ & $6(26.08 \%)$ & 0.85 \\
\hline USG for PCOS features & 1.00 \\
\hline 1. Present & $19(82.60 \%)$ & $19(82.60 \%)$ & \multirow{2}{|c|}{$4(17.39 \%)$} \\
\hline 2. Absent & $4(17.39 \%)$ & \\
\hline $\begin{array}{l}\text { BMI: body mass index; FG score: modified Ferriman-Gallwey score; } \\
\text { USG: ultrasonography; PCOS: polycystic ovary syndrome }\end{array}$ \\
\hline
\end{tabular}


Table 2. Comparison of efficacy of metformin and oral contraceptive containing drospirenone on body mass index, serum testosterone and DHEAS levels at baseline, 3 and 6 months of treatment

\begin{tabular}{|c|c|c|c|c|c|c|c|c|c|}
\hline \multirow[t]{2}{*}{ Parameters } & \multicolumn{3}{|c|}{$\begin{array}{c}\text { Baseline } \\
(\text { Mean } \pm \text { SD) }\end{array}$} & \multicolumn{3}{|c|}{$\begin{array}{c}3 \text { months of therapy } \\
(\text { Mean } \pm \text { SD) }\end{array}$} & \multicolumn{3}{|c|}{$\begin{array}{c}6 \text { months of therapy } \\
(\text { Mean } \pm \text { SD) }\end{array}$} \\
\hline & Met & Drosp & $\mathbf{p}$ & Met & Drosp & $\mathbf{p}$ & Met & Drosp & $\mathbf{p}$ \\
\hline $\begin{array}{l}\text { Serum Testoster- } \\
\text { one }(n g / d L)\end{array}$ & $\begin{array}{c}56.52 \pm \\
11.70\end{array}$ & $\begin{array}{c}62.23 \pm \\
13.59\end{array}$ & 0.15 & $\begin{array}{l}51.75 \pm \\
10.43^{* * *}\end{array}$ & $\begin{array}{l}53.94 \pm \\
15.40 * * *\end{array}$ & 0.68 & $\begin{array}{l}49.67 \pm \\
13.68 * *\end{array}$ & $\begin{array}{l}52.57 \pm \\
14.17 * * *\end{array}$ & 0.65 \\
\hline & & & \multicolumn{2}{|c|}{$\begin{array}{l}\text { SD: standard deviation; Met: metformin; Drosp: oral contraceptive containing drospirenone; BMI: body mass index; DHEAS: dehydroepian- } \\
\text { drosterone sulphate }\end{array}$} & $\begin{array}{l}\text { pirenone; } \mathrm{B} \\
\text { tively withir } \\
\text { n baseline }\end{array}$ & & $\begin{array}{l}\text { index; DHE } \\
\text { group (Me } \\
\text { hs of treatn }\end{array}$ & $\begin{array}{l}\text { S: dehydro } \\
\text { ersus Met; } \\
\text { nt. }\end{array}$ & $\begin{array}{l}* * * \text { and } * * * \text { denote statistical significance at } \mathrm{p}<0.05, \mathrm{p}<0.01 \text { and } \mathrm{p}<0.001 \text { respectively within the individual group (Met versus Met; Drosp } \\
\text { versus Drosp) for decline in BMI, serum testosterone and serum DHEAS levels from baseline at } 3 \text { and } 6 \text { months of treatment. }\end{array}$ \\
\hline
\end{tabular}

the two groups. Bobde et al. (10) reported a significant regularization of menstrual cycle in patients who received oral contraceptive containing drospirenone compared with those who received metformin $(p=0.001)$. This is also reported in a review by Costello et al. (11), where 18 of 21 patients on metformin and 20 of 24 patients on oral contraceptives had oligomenorrhea. Metformin was less effective compared with oral contraceptives in improving menstrual pattern $(p=0.004)$. In this study, the results observed at 3 months are consistent with earlier studies. The mechanism for regularization of cycles in oral contraceptive containing drospirenone users is withdrawal bleeding that occurs because of the hormone-free interval in the monthly cycle. In this study, the delayed effect of metformin on the menstrual cycle is possibly because of the lag period of 4-6 months that is required for the plasma luteinizing hormone ( $\mathrm{LH}$ ) levels to return to normal following decreased LH pulse secretion when the patient is initiated on metformin therapy (10).

There was no significant difference in BMI observed between the two groups at 6 months. Cinar et al. (12) reported no decline in BMI among 25 patients who received oral contraceptive containing drospirenone alone, whereas a decline in BMI was observed among 20 patients who received metformin along with an oral contraceptive. In another study by Ibáñez et al. (13), oral contraceptive containing drospirenone alone was ineffective in reversing dysadipocytokinemia, hypertriglyceridemia, and adiposity, whereas patients who received add-on metformin demonstrated reversal of the aforementioned features. Weight loss because of metformin observed in this study could be because of its anorexic property and its ability to counteract adipose tissue expansion through direct inhibition of adipogenesis (14). Moreover, Oelkers et al. (15) reported reduction in body weight because of its anti-mineralocorticoid properties of drospirenone in healthy young menstruating women who received oral contraceptive containing drospirenone. The results of this study re-instate that weight loss is observed with oral contraceptive containing drospirenone. The problem of weight gain experienced with the use of conventional combined oral contraceptives is, thus, reduced with oral contraceptives containing drospirenone.

In this study, no significant difference is observed between the two groups in decreasing serum testosterone and DHEAS levels. A review by Jakubowicz et al. (16) reported a $44 \%$ decrease in serum androgens in obese women diagnosed with PCOS. A decline in serum androgens is also reported in lean women diagnosed with PCOS who were on metformin therapy. However, Bobde et al. (10) and Cosma et al. (17) reported oral contraceptive containing drospirenone to be more effective in improving hirsutism in patients with PCOS. Drospirenone being a 17- $\alpha$ spironolactone derivative exhibits anti-androgenic property. This property is also exhibited by metformin in this study probably because of its ability to treat insulin resistance in this inherently insulin-resistant population. Insulin resistance is postulated as a mechanism in the pathogenesis of PCOS $(18,19)$ because of the following hypotheses. First, hyperinsulinemia is hypothesized to increase ovarian testosterone production by stimulating insulin receptors present on the ovarian theca cells, which act by a signaling pathway different from that mediating the metabolic effects of insulin. Second, hyperinsulinemia results in decreased synthesis of sex hormone-binding globulin in the liver, and thus, free testosterone levels are increased. Third, insulin facilitates local activity of insulin-like growth factor-binding protein-1 (IGF-1) in the ovary, which stimulates ovarian androgen production. Both the drugs were well tolerated with no serious adverse events being recorded.

The results of this study suggest that the universal guidelines released by the Endocrine society may not be applicable to this population; hence, ethnicity-wise modifications may be required. Good response to metformin on hyperandrogenism in this patient subset proves that it may be beneficial for South Asians who are more insulin resistant compared with Caucasians. Further studies are required.

The limitations of the study are that the study design was observational, and there was loss to follow-up of patients. Li et 
al. (20) also reported a 25.6\% treatment compliance and followup rate among patients with PCOS in the 6-month study period. Non-compliance and subsequent failure to return for review was observed among patients with an increased BMI, and the contributing factors included the long duration of therapy, delayed beneficial effects of drug treatment, and concern regarding adverse drug reactions. This study depicts a similar scenario, and thus, reinforces the need to develop educational tools and materials to strengthen treatment education and intervention in the clinic. Moreover, drug treatment for patients with PCOS is provided on an outpatient basis. In this age of advancing technology, the development of mobile applications to provide reminders to patients to take their pill daily at home and go to the clinic for regular reviews can be a simple, yet effective step to ensure treatment compliance.

Ethics Committee Approval: Ethics committee approval was received for this study from the ethics committee of M.S. Ramaiah Medical College and Hospitals.

Informed Consent: Informed consent was obtained.

Peer-review: Externally peer-reviewed.

Author Contributions: Concept - Y.S., N.M., P.K.; Design - Y.S., N.M., P.K.; Supervision - M.C.S.; Materials - P.K.; Data Collection and/or Processing - Y.S.; Analysis and/or Interpretation - Y.S., N.M.; Literature Review - Y.S.; Writer - Y.S.; Critical Review - N.M., P.K.; Other - M.C.S.

Conflict of Interest: No conflict of interest was declared by the authors.

Financial Disclosure: The authors declared that this study has received no financial support.

\section{References}

1. Sirmans SM, Pate KA. Epidemiology, diagnosis, and management of polycystic ovary syndrome. Clin Epidemiol 2014; 6: 1-13.

2. Boyle J, Terde HJ. Polycystic Ovary Syndrome An update. Reprod Health 2012; 41: 752-6.

3. Bozdag G, Yildiz BO. Combined oral contraceptives in polycystic ovary syndrome - indications and cautions. Front Horm Res 2013; 40: 115-27. [CrossRef]

4. Lashen H. Role of metformin in the management of polycystic ovary syndrome. Therapeutic Advances in Endocrinology and Metabolism 2010; 13: 117-28. [CrossRef]

5 . Johnson NP. Metformin use in women with polycystic ovary syndrome. Ann Transl Med 2014; 2: 56.

6. Legro RS, Arslanian SA, Ehrmann DA, Hoeger KM, Murad MH, Pasquali R, et al. Diagnosis and treatment of polycystic ovary syndrome: an endocrine society clinical practice guidelines. J Clin Endocrinol Metab 2013; 98: 4565-92. [CrossRef]

7. Deepa R, Shanthirani CS, Premalatha G, Sastry NG, Mohan V. Prevalence of insulin resistance syndrome in a selected south
Indian population--the Chennai urban population study-7 [CUPS7]. Indian J Med Res 2002; 115: 118-27.

8. Wijeyaratne CN, Balen AH, Barth JH, Belchetz PE. Clinical manifestations and insulin resistance (IR) in polycystic ovary syndrome (PCOS) among South Asians and Caucasians: is there a difference? Clin Endocrinol (Oxf) 2002; 57: 343-50. [CrossRef]

9. Soper DS. A-priori Sample Size Calculator for Student t-Tests [Software]. Available from: http://www.danielsoper.com/statcalc

10. Bobde JA, Bhosle D, Kadam R, Shelke S. Comparison of efficacy and safety of metformin, oral contraceptive combination of ethinyl estradiol and drospirenone alone or in combination in polycystic ovarian syndrome. J Obes Metab Res 2014; 1: 112-7. [CrossRef]

11. Costello M, Shrestha B, Eden J, Sjoblom P, Johnson N. Insulin-sensitising drugs versus the combined oral contraceptive pill for hirsutism, acne and risk of diabetes, cardiovascular disease, and endometrial cancer in polycystic ovary syndrome. Cochrane Database Syst Rev 2007; 1: CD005552. [CrossRef]

12. Cinar N, Harmanci A, Bayraktar M, Yildiz BO. Ethinyl estradiol-drospirenone vs ethinyl estradiol-drospirenone plus metformin in the treatment of lean women with polycystic ovary syndrome. Clin Endocrinol (Oxf) 2013; 78: 379-84. [CrossRef]

13. Ibáñez L, de Zegher F. Ethinylestradiol-drospirenone, flutamide-metformin, or both for adolescents and women with hyperinsulinemic hyperandrogenism: Opposite effects on adipocytokines and body adiposity. J Clin Endocrinol Metab 2004; 89: 1592-7. [CrossRef]

14. Alexandre KB, Smit AM, Gray IP, Crowther NJ. Metformin inhibits intracellular lipid accumulation in the murine pre-adipocyte cell line, 3T3-L1. Diabetes Obes Metab 2008; 10: 688-90. [CrossRef]

15. Oelkers W, Foidart JM, Dombrovicz N ,Welter A, Heithecker R. Effects of a new oral contraceptive containing an antimineralocorticoid progestogen, drospirenone, on the renin - aldosterone system, body weight, blood pressure, glucose tolerance and lipid metabolism. J Clin Endocrinol Metab 1995; 80: 1816-21. [CrossRef]

16. JakubowiczD, Seppala M. Effects of Metformin on Hyperinsulinemia, Hyperandrogenism and Reproduction in Women with Polycystic Ovarian Syndrome. J Endocrinol Diabetes Obes 2014; 2: 1034.

17. Cosma M, Swiglo BA, Flynn DN, Kurtz DM, Labella ML, Mullan RJ, et al. Clinical review: Insulin sensitizers for the treatment of hirsutism: A systematic review and metaanalyses of randomized controlled trials. J Clin Endocrinol Metab 2008; 93: 1135-42. [CrossRef]

18. Rojas J, Chávez M, Olivar L, Rojas M, Morillo J, Mejías J, et al. Polycystic Ovary Syndrome, Insulin Resistance, and Obesity: Navigating the Pathophysiologic Labyrinth. Int J Reprod Med 2014; 2014: 719050. [CrossRef]

19. Dunaif A. Insulin resistance and the polycystic ovary syndrome: Mechanism and implications or pathogenesis. Endocr Rev 1997; 18: 774-800. [CrossRef]

20. Li S, He A, Yang J, Yin T, Xu W. A logistic regression analysis of factors related to the treatment compliance of infertile patients with polycystic ovary syndrome. J Reprod Med 2011; 56: 325-32. 